\title{
Maternidade em Idade Avançada: Aspectos Teóricos e Empíricos
}

\author{
Aline Grill Gomes \\ Tagma Marina Schneider Donelli \\ Cesar Augusto Piccinini \\ Rita de Cássia Sobreira Lopes \\ Universidade Federal do Rio Grande do Sul
}

\begin{abstract}
RESUMO
O presente artigo apresenta uma revisão da literatura sobre o tema da maternidade em idade avançada, examinando os aspectos teóricos e empíricos da gestação após os 35 anos, tanto sob a perspectiva médica quanto psicológica. De modo geral, a medicina considera esta idade como ponto de corte para incluir as gestantes em um grupo de alto risco, prevendo uma série de complicações possíveis tanto para o bebê como para a mulher. No entanto, sabe-se que existem outros fatores capazes de intensificar ou amenizar o risco de uma gestação a partir desta idade. No contexto da maternidade após os 35 anos, é importante que os profissionais da saúde orientem-se por uma abordagem que transcenda o critério exclusivo da idade, e considerem os diversos fatores envolvidos no desenrolar de uma gestação segura e tranqüila, tais como as condições clínicas de mãe e bebê, e as condições emocionais da mãe para lidar com as demandas emocionais provocadas pela gravidez.
\end{abstract}

Palavras-chave: maternidade em idade avançada; gestação; alto risco.

\begin{abstract}
Delayed Motherhood: Theoretical and Empirical Aspects

The present article presents a literature review on delayed motherhood. It examines the theoretical and empirical aspects of pregnancy after 35 years, both from the medical and psychological perspective. In general, medicine considers this age as a cut-off point for including pregnant women within high-risk groups, foreseeing a series of possible complications both for the baby and for the mother. However, it is known that other factors exist which are able to heighten or reduce the risk of a pregnancy beginning at this age. In the context of motherhood after 35 , it is important that health professionals be guided by an approach that transcends the exclusive criterion of age, and consider the several factors involved in a safe and calm pregnancy, such as the clinical and psychological conditions of the woman, as well as the conditions for dealing with the emotional demands of pregnancy.
\end{abstract}

Keywords: delayed motherhood; pregnancy; high risk.

A gravidez é um período marcado por mudanças de diversas ordens, além de representar para a mulher uma experiência única, repleta de sentimentos e emoções de muita intensidade (Brazelton \& Cramer, 1992; Klaus \& Kennell, 1993; Raphael-Leff, 1997, 2000; Soifer, 1980). Gestar é mais do que possibilitar o crescimento e o desenvolvimento fetal; envolve uma adaptação biológica, corporal e também psíquica. É um momento de reconfiguração de relações e reordenamento do espaço psicológico interno da mulher (Rubin, 1975).

Nessa transição, especialmente as primíparas precisam passar da condição de somente filha para tam- bém mãe, reviver experiências anteriores, além de precisar reajustar seu relacionamento conjugal e sua situação sócio-econômica (Maldonado, 1997). Diante de todas estas mudanças e revivências, a experiência de gestar faz com que a mulher tenha sua sensibilidade aguçada, o que a torna, muitas vezes, suscetível a distúrbios emocionais (Raphael-Leff, 2000). É o que Bydlowski (1997) chama de "transparência psíquica", como sendo um estado próprio deste momento, no qual a mulher experimenta sentimentos intensos e revive profundamente a relação com a própria mãe. A partir desse jogo de identificações vai, então, se construindo um espaço psíquico para o seu bebê. 
Assim, a gravidez pode tanto desencadear uma crise emocional com um desfecho patológico para as gestantes, como inaugurar um potencial de adaptação e resolução de conflitos, até então desconhecido (Bibring \& Valenstein, 1976; Leifer, 1977; Maldonado, 1997). O resultado deste processo poderá influenciar fortemente a relação futura com a criança (Caron, 2000; Maldonado, 1997).

Inúmeros fatores de ordem intrapsíquica e contextual tendem a influenciar o andamento da gravidez, tais como a estrutura de personalidade da gestante, o nível de resoluções de seus conflitos, e o suporte familiar que ampara a mulher durante a gestação (Bibring, Dwyer, Huntington \& Valenstein, 1961). Soifer (1980) acrescenta, ainda, os efeitos do estado psicológico atual da gestante, as condições do seu vínculo conjugal, sua aceitação de ter um filho e sua condição econômica. Os objetos internos da mulher influenciam, então, sua capacidade de engravidar, manter a gravidez e dar à luz, isto é, têm grande poder no percurso da maternidade (Bradley, 2000).

Dentro deste contexto, as ansiedades e as inadaptações são comuns ao processo gravídico normal. A gravidez é por si só um desafio adaptativo, pois implica mudanças que atingem a mulher nos seus aspectos fisiológico, psicológico e social (Tedesco, 1997). Segundo o autor, ela experimenta um desequilíbrio de tal ordem que a gravidez é sentida como um grande evento estressor. Por isso, Isfer (1997) justifica que toda gravidez é considerada, em alguma medida, de risco, mesmo que de baixo risco.

Do ponto de vista médico, alguns fatores são responsáveis por configurar uma gestação como de alto risco, os quais podem ser divididos em dois grupos: os fatores de risco presentes já antes da gestação e os que surgem durante este período (Isfer, 1997). Os primeiros incluem idade da mãe, doença materna, antecedentes obstétricos, origem étnica, história familiar de doenças hereditárias, e problemas de consangüinidade. Os fatores que surgem durante a gravidez correspondem, entre outros, à alfa-feto-proteína materna demasiadamente elevada ou baixa, à infecção materna, à hipertensão, ao diabetes gestacional, à exposição da mãe a agentes teratogênicos e/ou a radiação, e a alterações ecográficas. A ocorrência de algum destes fatores intensifica as angústias e as dificuldades próprias da gestação: o temor da mulher em relação a si própria e ao feto passam a ser reais (Tedesco, 1997).

Já do ponto de vista emocional, é necessário o cumprimento de algumas tarefas durante o período gestacional, as quais vão desde a aceitação da gravidez, o reconhecimento do bebê e o estabelecimento de um vínculo de afeto com ele, até a preparação para a separação mãe-bebê por ocasião do parto (Missonier $\&$ Solis-Ponton, 2004). Sabe-se que as eventuais dificuldades de cumprimento dessas tarefas e de adaptação da gestante às mudanças psicológicas exigidas neste período podem incrementar a ansiedade materna e prejudicar tanto o andamento como o desfecho da gestação.

Gravidez de alto risco pode ser entendida, então, como aquela que apresenta maior probabilidade de ter evolução desfavorável, seja para o feto, seja para a mãe (Buchabqui, Abeche \& Brietze, 2001). Para estes autores, o que se denomina como fatores de risco inclui circunstâncias ou características que se associam à maior probabilidade de ocorrer dano à saúde do feto e/ou da mãe. A gestação rotulada como de alto risco tende a incrementar as dificuldades de uma gravidez normal, na medida em que introduz para a gestante uma preocupação real em relação às suas condições de saúde, bem como de seu bebê (Tedesco, 1997). Assim, segundo o autor, a gravidez de alto risco pode representar, tanto para a gestante como para a equipe médica, um indicativo de maiores problemas emocionais e sociais associados à vivência desse período da vida. Por estas razões, a gestante incluída em um grupo de gravidez de alto risco tende a se sentir mais frágil, apreensiva e impotente. Associado a isto ocorre um incremento dos níveis de ansiedade materna.

Especialmente em relação à ansiedade materna, podem ser citadas algumas repercussões no ciclo gravídico-puerperal, tais como o aparecimento de problemas relacionados com a evolução da gravidez, com o parto e com o relacionamento posterior com o bebê. Por exemplo, as mulheres que apresentam mais sintomas de tensão muscular durante a gestação do que a média, e aquelas que demonstram, no início do trabalho de parto, mais sinais psicológicos e comportamentais associados à ansiedade, são mais propensas a apresentarem distúrbios fisiológicos relacionados com a disfunção uterina (Petrie \& Williams, 1996). Da mesma forma, níveis intensos de ansiedade e de medo interferem nitidamente na contratilidade uterina, alterando o ritmo e a força das contrações do trabalho de parto (Petrie \& Williams, 1996; Maldonado, 1997). Além disso, os escores de Apgar do recém-nascido tendem a ser mais baixos nos filhos de gestantes que apresentaram alto grau de ansiedade (Petrie \& Williams, 1996). Outro estudo referido por Maldonado (1997) também aponta uma diminuição significativa do 
Apgar de bebês cujas mães eram altamente ansiosas, em comparação com mães menos ansiosas.

Dentre os fatores de risco anteriormente citados, o presente estudo destaca a idade materna, e a inclusão das gestantes com mais de 35 anos em um grupo de alto risco, o que tende a exacerbar as dificuldades de uma gestação "normal" e, conseqüentemente, elevar os níveis de ansiedade materna. A revisão da literatura examinada a seguir busca explorar tanto os aspectos histórico-sociais da maternidade após esta idade, bem como os seus aspectos médicos e emocionais.

\section{Maternidade após os 35 anos}

Atualmente, tem se observado um aumento do número de gestações acima dos 35 anos, especialmente em países industrializados ${ }^{1}$ (Blickstein, 2003; Postmontier, 2002; Windridge \& Berryman, 1999). Entre as razões para tal tendência encontram-se a ampla disponibilidade de métodos contraceptivos, a postergação do matrimônio, a maior incidência de divórcios, o desejo de atingir um nível educacional e profissional mais elevado, de conquistar estabilidade e independência financeira, e o aprimoramento de técnicas de fertilização artificial (Tarin, Brines \& Cano, 1998; Zavaschi, Costa, Brunstein, Kruter \& Estrella, 1999). Entretanto, a maternidade concretizada no limite ou mesmo após essa idade é, hoje, uma nova realidade, que se deve, principalmente, aos avanços da medicina e à implementação de técnicas de reprodução assistida, o que vem fazendo com que não exista mais uma fronteira precisa entre gestantes jovens e com mais idade (Blickstein, 2003).

Após os 35 anos, ocorre uma diminuição da capacidade reprodutiva, pois as células sexuais femininas começam a escassear ocorrendo um declínio da fertilidade (Versellini, Zuliane, Rognoni, Trespidi, Oldani $\&$ Cardinale, 1992). Sabe-se que após essa idade aumenta a incidência de abortos, anormalidades fetais, diabetes, hipertensão, hemorragias, partos prematuros, trabalho de parto disfuncional, partos por cesariana, baixo peso do bebê ao nascimento, maiores índices de sofrimento fetal, e menores índices de Apgar do bebê no quinto minuto (Milner, Barry-Kinsella, Unwin \& Harrison, 1992; Postmontier, 2002; Versellini \& cols., 1992).

Dessa forma, a gravidez após esta idade tende a ser considerada como tardia no meio médico, o que confere a estas gestantes o estigma de grávidas idosas, maternidade avançada, maternidade atrasada, mães "inapropriadas" e até grávidas pré-menopáusicas
(Milner \& cols., 1992; Montgomery, 1997; Woollett \& Marshall, 2001). Fica claro, então, que a idade materna, por si só, vem sendo destacada muitas vezes como um fator de risco para a gravidez, o que faz com que as mulheres com mais de 35 anos sejam tratadas com um manejo especial desde o início da sua gestação. Tal manejo diferenciado pressupõe uma indicação de maior número de consultas e variedade de procedimentos pré-natais, dentre os quais se ressaltam os invasivos tais como a biópsia de vilosidades coriônicas e a amniocentese.

$\mathrm{Na}$ verdade, do ponto de vista emocional, os autores relatam tanto uma postura facilitadora quanto dificultadora do processo de transição para a maternidade de grávidas após os 35 anos. A primeira se evidenciaria através da maior maturidade (Postmontier, 2002), melhores condições de saúde mental (Windridge \& Berryman, 1999) e menor medo de ficar sem ajuda e de perder o controle durante o parto (Stark, 1997). Ao mesmo tempo, elas tendem a culpar menos freqüentemente a equipe médica pela dor do parto e a ficar mais satisfeitas com a assistência recebida (Windridge \& Berryman, 1999). DeVore (1983) também identificou algumas vantagens psicológicas das gestantes com idade avançada, entre elas a experiência de vida e uma identidade mais consolidada, o que faz com que estejam melhor preparadas para aceitar o bebê como um indivíduo separado delas e com características próprias, promovendo com mais êxito o seu desenvolvimento emocional.

Por outro lado, a postura dificultadora se manifesta, entre outras formas, através da disposição física reduzida (DeVore, 1983), menos atitudes positivas em relação à maternidade e mais ansiedade em relação ao cuidado com a criança no período pós-parto (Reece, 1993). Destaca-se, também, a percepção constante de que o seu bebê corre risco durante a gravidez e o parto, ou que ele seja mais vulnerável, mesmo sem que a gestante tenha apresentado qualquer problema de saúde ou complicação durante a gestação (Windridge \& Berryman, 1999). Tal percepção pode evocar, segundo esses autores, mais sentimentos de culpa nas mulheres que adiaram a gestação, acreditando que é exclusivamente sua a responsabilidade pelas condições de saúde do bebê. Além disso, Randell (1993) encontrou maior resistência a mudanças e maiores problemas de adaptação à maternidade em gestantes com mais de 35 anos. Contudo, alguns poucos autores encontraram dados opostos aos descritos acima mostrando que as mulheres mais jovens é que apresentavam mais dificuldades na maternidade quando comparadas às com 
idade mais avançada (Gottesman, 1992; Robinson, Garner, Gare \& Crawford, 1987).

Uma análise da literatura acerca da influência de vários fatores sobre o curso e o desfecho da gestação após os 35 anos, permitiu organizar os estudos em dois grupos diferentes. O primeiro prioriza a variável idade em relação a outros fatores, tais como condições prévias de saúde, acesso ao sistema de saúde, nível sócio-econômico e educacional e características de personalidade. Já o segundo grupo tende a uma postura mais relativista, equiparando a influência destes outros fatores com a idade. Além disso, encontram-se estudos que apontam a importância de intervenção psicológica na gestação após esta idade.

Especificamente em relação ao primeiro grupo, destaca-se a revisão de Tarin, Brines e Cano (1998) que procuraram explicar quais as possíveis conseqüências da postergação da maternidade sobre os filhos. Eles enfatizaram os estudos que apontam para os prejuízos, diretos ou indiretos, que a idade materna e paterna avançada pode trazer para a prole. Os autores destacaram o aumento das complicações obstétricas e/ou problemas fetais e perinatais, os quais poderiam aumentar o risco de mortalidade e morbidade em recém-nascidos e crianças no decorrer da vida. Também foram citadas associações com paralisia cerebral, epilepsia, autismo, dislexia, psicose e disfunções na motricidade fina, percepção visual e déficit de atenção. Especificamente na mulher identifica-se menor probabilidade de filhos do sexo masculino e maior probabilidade de conceber um bebê com trissomia do cromossomo 21, e/ou com desordens mitocôndricas do DNA. No homem, aumentam, com a idade, as chances de ter filhos que apresentam desordens hereditárias.

Em um estudo empírico posterior (Tarin, Vidal, Perez-Hoyos, Cano \& Balasch, 2001), 93 indivíduos inférteis foram solicitados a preencher um questionário que abordou questões sobre histórias de infertilidade na família, possíveis causas da infertilidade e condições de saúde e idade dos pais no momento do seu nascimento. Os resultados demonstraram que quanto mais avançada a idade materna na época do parto maior era a probabilidade de os filhos homens serem inférteis. Tais achados direcionaram os autores a serem taxativos ao afirmar que a postergação da maternidade tenderia a aumentar a probabilidade de infertilidade nos filhos, e, por isto, deveria ser evitada.

Ainda tratando-se do primeiro grupo de estudos, Francis (1985) se mostrou incisivo ao afirmar que a gravidez seria ideal em mulheres entre 20 e 30 anos, tolerável entre 30 e 34, indesejável entre 35 e 39, devendo ser evitada após os 40 anos. O autor chegou a essa indicação através de um estudo que coletava os dados nos registros médicos de pacientes obstétricas. Nesta mesma direção o estudo de Milner, BarryKinsella, Unwin e Harrison (1992) comparou 35 variáveis de significância clínica em dois grupos: um de mulheres com 40 anos ou mais e outro de mulheres com menos de 40 anos. Os dados foram coletados e analisados retrospectivamente. $\mathrm{O}$ grupo com mais idade apresentou aumentos significativos em diabetes gestacional, hemorragia pré-parto, sofrimento fetal, prematuridade, baixo peso ao nascer e mortalidade perinatal, anormalidades cromossômicas congênitas (especialmente Síndrome de Down), além de taxas aumentadas de parto induzido e cesário. Os autores reconheceram que podem existir outros fatores, além da idade, igualmente importantes para incluir estas gestantes num grupo de alto risco. Porém, a dificuldade de delimitar a influência de cada um destes fatores isoladamente sobre os resultados obstétricos levou-os a recomendar que grávidas nesta faixa etária, chamadas por eles de "grávidas idosas", devessem ser consideradas como de alto risco, merecendo manejo diferenciado por parte da equipe de saúde.

Já o segundo grupo de estudos apresenta uma postura mais relativista, como exemplificado na revisão da literatura realizada por Blickstein (2003). Embora a autora aponte para o maior risco de complicações em uma gravidez depois dos 35 anos, ressalta que tais complicações devem ser entendidas também com base em outros fatores, tais como o resultado cumulativo de doenças prévias. Blickstein é enfática ao afirmar que não há evidências diretas de que a idade materna, por si só, seja um complicador da gestação ou do parto. Ao mesmo tempo, os resultados perinatais de gestantes nessa faixa etária são melhores quando se trata de gêmeos e trigêmeos. A autora também acredita que as intervenções junto a grávidas com mais de 35 anos devem levar em consideração outros aspectos além da idade. Assim, enfatiza que a equipe de saúde e o manejo médico devem procurar se afastar do viés da idade como fator preponderante nas complicações de uma gestação nessa faixa etária.

Destaca-se, também, dentro deste mesmo grupo, o estudo de Reece (1993), que investigou, longitudinalmente, entre outros fatores, o apoio social e primeira experiência de maternidade em 91 primíparas com mais de 35 anos. Os dados foram coletados no último trimestre de gestação e um mês após o parto. Segundo a autora, o apoio social inadequado, a alta exigência 
sobre si mesmas como mães e uma baixa satisfação com a parentalidade justificariam a necessidade de intervenção. O estudo de Reece destaca-se pela ênfase em variáveis sociais e emocionais, em detrimento de aspectos médicos explorados por outros estudos. Nesse sentido, o artigo em questão, apesar de não ser um estudo comparativo, evita tomar a idade, por si só, como uma variável mais importante que as outras envolvidas em uma gravidez tardia. $\mathrm{O}$ estudo sugere priorizar o aconselhamento individual, a provisão de informações antecipatórias sobre a parentalidade, além de uma facilitação para o desenvolvimento de uma rede de apoio, evidenciando sua preocupação com as particularidades de cada gestação.

Também para Harker e Thorpe (1992) tanto fatores biológicos como psicológicos devem ser examinados conjuntamente, para determinar o curso de uma gravidez e a adaptação à maternidade após 35 anos. Os autores entendem que se trata de elementos compensatórios e complementares, trazendo uma compreensão mais ampla da gestante e de sua possível inclusão em um grupo de gravidez de alto risco. Além deles, Milner e cols. (1992) enfatizaram que as intervenções para grávidas de alto risco devem ter como base uma abordagem individual, levando em consideração os aspectos emocionais, culturais, sociais e também biológicos pertinentes a cada caso.

Ainda adotando uma postura relativista, Windridge e Berryman (1999) publicaram um estudo que comparou experiências de gravidez, parto e do primeiro ano da maternidade de 54 mulheres com idade superior a $35 \operatorname{anos}(\mathrm{M}=37$ anos $)$, divididas em igual número entre primíparas e multíparas, e 53 mulheres entre 20 e 29 anos, sendo 26 delas primíparas e 27 multíparas. As mulheres com mais idade tiveram mais probabilidade de ser classificadas como tendo gravidez de alto risco (tal classificação estava codificada nas anotações e não foi tornada do conhecimento das mulheres). Elas também fizeram mais exames de ultrassom e amniocenteses. A primiparidade, e não a idade, esteve associada com o uso de maior número de intervenções no parto vaginal. Mortalidade perinatal e outros dados obstétricos não apareceram associados à idade. Segundo o estudo, as experiências das mulheres mais velhas foram mais positivas que as do grupo mais jovem, pois se diziam mais felizes. Além disso, segundo os autores, as mulheres com 35 anos ou mais, não teriam razões objetivas para possuírem sérias preocupações com a segurança de seus bebês ou para ter prejuízos emocionais no pós-parto. Porém, o fato de se incluir as gestantes com 35 anos ou mais em um grupo de risco as faz ter mais preocupações com a segurança do bebê, o que poderia acabar afetando negativamente a interação mãe-feto.

Outro estudo que destacou esta idéia foi realizado por DeVore (1983), o qual apontou que, por anos, a obstetrícia considerou a mulher de 35 anos ou mais que estava tendo seu primeiro bebê como uma primigesta idosa, de idade avançada ou ultrapassada e, supostamente, uma paciente de alto risco. Segundo o estudo, os riscos maternos de uma gestação em idade avançada dizem respeito, especialmente, a problemas pré-existentes, que estão associados com o avanço da idade (diabetes, hipertensão, problemas cardiovasculares, doença renal e fibrose uterina). O autor aponta algumas vantagens psicológicas da maternidade após os 35 anos, pois os pais mais velhos tendem a estar melhor preparados para cuidar de uma criança por sentirem-se comumente melhores consigo mesmos, serem mais tolerantes, aceitarem melhor a criança como um indivíduo e por terem melhores condições para o desenvolvimento de suas potencialidades como genitores. Além disso, podem decidir de forma mais consciente pela parentalidade e possuem melhores condições econômicas.

Postmontier (2002) também apontou algumas vantagens em adiar a maternidade. A segurança financeira e a maior maturidade podem garantir uma melhor qualidade à parentalidade. Ao mesmo tempo, em função do aumento no número de mulheres que estão tendo filhos com mais de 35 anos, a autora concluiu, em seu artigo de revisão da literatura, que se faz necessária uma mudança nos cuidados pré-natais oferecidos, levando em conta essa nova realidade.

O estudo de Gottesman (1992) comparou três grupos de gestantes: um de mulheres com idades entre 20 e 24 anos, outro entre 25 e 29 anos, e o último com idades superiores a 30 anos. Foram utilizadas entrevistas para investigar a relação materno-fetal, a qualidade do relacionamento conjugal, a preparação para a maternidade, as atitudes em relação à gravidez, o conceito de papel materno e a motivação para a maternidade. $\mathrm{O}$ estudo revelou que as mulheres mais jovens experimentaram mais dificuldades de ajustamento ao papel materno durante a gestação, em comparação com os dois grupos mais velhos. Já os grupos de mulheres com idades entre 25 e 29 anos, e com mais de 30 anos, se mostraram muito semelhantes. As variáveis mais associadas ao grupo de mulheres com mais de 30 anos foram uma maior motivação para a maternidade, uma melhor relação materno-fetal e um melhor conceito sobre o papel materno. O estudo afirmou 
ainda que a gravidez é um período intenso de preparação, tanto da mente quanto do corpo, e destacou que os cuidados pré-natais precisariam incluir uma abordagem individualizada e sensível que fizesse frente também aos aspectos emocionais desse processo.

Por fim, pode ser ainda citado neste grupo de estudos o realizado por Winslow (1987), que investigou como as mulheres com idade avançada vivenciam a maternidade. Para tanto entrevistou 12 mulheres primíparas, entre 35 e 44 anos, com cônjuges entre 31 e 43. A análise das entrevistas evidenciou que as mulheres desta faixa etária tendiam a ter uma vivência particular da maternidade. Dentre os aspectos salientados no estudo destaca-se a satisfação por terem suas vidas melhor organizadas, julgando como sendo, então, "o momento certo". Em contrapartida, elas também salientaram a dificuldade de enfrentar naquele momento uma vida tão nova e tão diferente daquela já estruturada, o que levou a autora a sugerir a possibilidade de intervenções junto a esta população.

Em relação às intervenções, o estudo de Milner e cols. (1992) destacou a importância da intervenção médica, recomendando incluir todas as gestantes com idade superior a 40 anos em um grupo de alto risco. Já o estudo de Reece (1993) sugeriu que a equipe de enfermagem realizasse intervenções que incluíssem aconselhamento individual para as mães, orientações para a parentalidade e desenvolvimento de grupos de apoio para ambos os genitores. Finalmente, Winslow (1987) indicou ainda a realização de intervenções psicoprofiláticas com mães de idade avançada. Estas agiriam no sentido de antecipar alguns problemas geralmente vivenciados por estas mães, principalmente relacionados às adequações da maternidade à vida pessoal e profissional.

\section{CONSIDERAÇÕES FINAIS}

A partir da revisão da literatura sobre a influência de diferentes fatores sobre o curso e o desfecho da gestação após os 35 anos, constata-se que parece não existir, entre os autores, um consenso de que a idade por si só constitui um determinante de futuros problemas no processo gravídico-puerperal. Entretanto, alguns autores ainda tendem a priorizar a variável idade que, isoladamente, é vista como responsável por problemas maternos e fetais. Esta visão determina posturas no manejo de muitos profissionais de saúde, que acabam enquadrando as mulheres com mais de 35 anos, apressada ou automaticamente, em um grupo de gestantes de alto risco.
É importante apontar que a própria definição de gravidez de alto risco acaba rotulando a gestante e trazendo-lhe sofrimento (Tedesco, 1997), antes mesmo que suas condições físicas, psicológicas e sociais sejam inteiramente examinadas. Ao ser considerada de alto risco reforça-se o rótulo de "ser diferente". A equipe médica, fazendo seu papel, tende a incrementar este sentimento, na medida em que concentra e direciona para a mulher atenção, cuidados e indicações específicas e, por vezes, até exacerbadas, fazendo com que ela fique bastante alerta para sua situação de risco. Esta situação pode contribuir para aflorar sentimentos como censura, culpa e incapacidade na gestante, que podem incrementar sua ansiedade e prejudicar a evolução normal deste período. O mecanismo da negação, quando extremado, pode até levar a gestante a evitar se relacionar com o bebê, para que não haja ligação afetiva com quem pode não sobreviver.

Além disso, o fato de ser colocada em um grupo de risco implica numa maior freqüência de consultas médicas, realização de infindáveis exames e procedimentos, bem como maior probabilidade de, por profilaxia, ser hospitalizada ou mantida em repouso. Esse tipo de conduta gera, inevitavelmente, aumento do nível de ansiedade materna. Conforme já referido, sabe-se que a ansiedade está associada ao aparecimento de problemas relacionados com a evolução da gravidez e com o parto, e tem importante impacto no relacionamento da mãe com o bebê, tanto na gestação como após o seu nascimento. Dessa forma, é preciso que se considere a ansiedade materna como mais um fator de risco importante na gestação em idade avançada e que se entenda que este pode ser exacerbado pelo manejo que se faz desta situação. Assim, cabe destacar a importância da sensibilidade da equipe médica a respeito das implicações psicológicas das diversas condutas que precisam ser eventualmente tomadas com estas gestantes, buscando minimizar o aumento de suas ansiedades e preocupações.

A literatura aponta que, de modo semelhante à idade materna avançada, a ansiedade da mãe também está associada a diversos problemas durante a gestação, no parto e para o próprio bebê. Assim, embora se constituam em dois fatores etiologicamente diferentes, a idade avançada e a ansiedade merecem ser consideradas conjuntamente. A forma como a equipe de saúde lida com as questões clínicas e obstétricas envolvendo a maternidade tardia deveria ser minuciosamente avaliada a fim de evitar que a gravidez após os 35 anos, que segundo a literatura já é uma situação potencialmente problemática, sofra ainda mais complicações em função da ansiedade aumentada. O manejo obsté- 
trico inadequado, a falta de sensibilidade dos profissionais de saúde e, de modo geral, o preconceito que cerca uma gravidez em idade avançada, pode ser fatores responsáveis pelo desencadeamento e pelo incremento da ansiedade materna.

Não se quer com isso reduzir a importância da idade materna avançada, que pode, obviamente, levar a complicações no ciclo gravídico-puerperal, especialmente quanto mais avançada ela for. Porém, do ponto de vista psicológico, esse fator por si só pode, eventualmente, aumentar os níveis de ansiedade das mães, ao se perceberem em um ponto de corte que as classifica como tendo uma gravidez de baixo ou alto risco, e sendo colocadas em um grupo com tratamento diferenciado. $\mathrm{O}$ que se sugere é que haja um minucioso trabalho de esclarecimento da equipe de saúde, evitando classificações apressadas e seus conseqüentes preconceitos, com destaque para uma avaliação global da saúde e qualidade de vida da gestante, que permitam pontuar, cuidadosamente, os eventuais riscos de determinada gestação tardia. Este trabalho pode, inclusive, não estar centrado na idade em si, mas em outros fatores como os destacados acima.

Assim, é importante que os profissionais da saúde se orientem por uma abordagem que transcenda o critério idade e considerem também os aspectos subjetivos, a história de vida e as condições sociais da gestante, reconhecendo que existe um funcionamento único que inclui aspectos físicos e emocionais. A gravidez em idade avançada deveria perder a conotação de "não natural", e passar a ser considerada como uma resultante de mudanças sociais e progressos médicos, merecendo maiores preocupações apenas depois de uma avaliação pormenorizada da complexidade de aspectos que podem estar associados às eventuais complicações. Isto exige que se tenha que abarcar os desafios e as complicações potenciais relativas à gestação em idade avançada que, atualmente, é cada vez mais comum do que no passado. Para tanto sugere-se que novos estudos sejam realizados enfocando as gestantes, as puérperas e os bebês de gravidezes tardias, para que seja possível propor intervenções mais adequadas a esse contexto.

É inegável que os cuidados médicos para as gestações de risco se tornaram mais eficientes no combate à mortalidade materna e perinatal e merecem todo nosso reconhecimento. Porém, estes avanços não incluíram uma melhora das condições psicológicas envolvendo essas situações (Tedesco, 1997). O diagnóstico de problemas clínicos e obstétricos, tanto maternos como fetais, vêm melhorando a cada dia, bem como a possibilidade da medicina de resolver os pro- blemas detectados. Entretanto, os aspectos emocionais ligados à manutenção do equilíbrio interpessoal e das relações familiares das gestantes ainda são pouco conhecidos, o que contribui para enfatizar o estigma e o preconceito em relação à maternidade em idade avançada. É necessário que os profissionais da saúde possam compreender e atuar com mais sensibilidade nessa situação, atentando para as particularidades de cada caso, e promovendo, assim, um estado de maior tranqüilidade emocional à dupla mãe-feto.

\section{REFERÊNCIAS}

Bibring, G., \& Valenstein, A. (1976). Psychological aspects of pregnancy. Clinical Obstetric and Gynecology, 19, 357-371.

Bibring, G. L., Dwyer, T. F., Huntington, D. S., \& Valenstein, A. F. (1961). A study of the psychological processes in pregnancy and of the earliest mother-child relationship. The Psychoanalytic Study of the Child, 16, 9-44.

Blickstein, I. (2003). Motherhood at or beyond the edge of reproductive age. International Journal of Fertility, 48(1), 17-24.

Bradley, E. (2000). Pregnancy and the internal world. Em J. Raphael-Leff (Org.). 'Spilt milk' perinatal loss \& breakdown (pp. 28-38). Londres: Institute of Psychoanalysis.

Brazelton, T. B., \& Cramer, B. G. (1992). As primeiras relações (M. B. Cipolla, Trad.). São Paulo: Martins Fontes.

Buchabqui, J., Abeche, A., \& Brietze, E. (2001). Assistência prénatal. Em F. Freitas, S. Martins-Costa, J. Ramos \& J. Magalhães (Orgs). Rotinas em obstetricia (pp. 23-37). Porto Alegre: Artes Médicas.

Bydlowski, M. (1997). La dette de vie. Paris: PUF.

Caron, N. (2000). O ambiente intra-uterino e a relação maternofetal. Em N. Caron (Org.), A relação pais-bebê: Da observação à clínica (pp. 119-134). São Paulo: Casa do Psicólogo.

DeVore, N. E. (1983). Parenthood postponed. American Journal of Nursing, 83(8), 1160-1163.

Francis, H. H. (1985). Delayed childbearing. IPPF Medical Bulletim, 19(3), 3-4.

Gottesman, M. M. (1992). Maternal adaptation during pregnancy among adult early middle, and late childbearers: Similarities and differences. Maternal-Child Nursing Journal, 20(2), 93110.

Harker, L., \& Thorpe, K. (1992). "The last egg in the basket?" elderly primiparity - A review of findings. Birth, 19(1), 23-30.

Isfer, E. (1997). Medicina fetal: O feto, o paciente. Em J. Wilheim (Org.). Decifrando a linguagem dos bebês - Anais do Segundo Encontro Brasileiro para o Estudo do Psiquismo Pré e Peri Natal (pp. 181-192). São Paulo: Cop L Print.

Klaus, M. H., \& Kennell, J. H. (1993). Pais-bebê: A formação do apego (D. Batista, Trad.). Porto Alegre: Artes Médicas.

Leifer, M. (1977). Psychological changes accompanying pregnancy and motherhood. Genetic Psychology Monographs, 95, 55-96.

Maldonado, M. T. P. (1997). Psicologia da gravidez, parto e puerpério (14 ${ }^{\mathrm{a}}$ ed.). São Paulo: Saraiva. 
Milner, M., Barry-Kinsella, C., Unwin, A., \& Harrison, R. F. (1992). The impact of maternal age on pregnancy and its outcome. International Journal of Ginecology and Obstetrics, 38, 281-86.

Missonier, S. \& Solis-Ponton, L. Parentalidad y embarazo. Convertirse em madre, convertirse em padre: Las interacciones entre los padres y su hijo antes del nacimiento. Em L. SolisPonton (Org.), La Parentalidad: Desafio para el tercer milênio, um homenaje internacional a Serge Lebovici. (pp. 75-82). Cidade do México: Editorial El Manual Moderno

Montgomery, M. (1997). Mecanismos psíquicos da gravidez tardia. Em M. Zugaib, J. J. Tedesco \& J. Quayle (Orgs.), Obstetrícia psicossomática. São Paulo: Atheneu.

Petrie, R. H., \& Williams, A. M. (1996). Trabalho de parto. Em R. A. Knuppel \& J. E. Drukker (Orgs.), Alto risco em obstetrícia: Um enfoque multidisciplinar (pp. 233-250). Porto Alegre: Artes Médicas.

Postmontier, B. E. (2002). Antepartum care in the twenty-first century. Nursing Clinic North America, 37, 757-769.

Randell, B. P. (1993). Growth versus stability: Older primiparous women as a paradigmatic case for persistence. Journal of Advanced Nursing, 18, 518-525.

Raphael-Leff, J. (1997). Gravidez: A história interior (R. D. Pereira, Trad.). Porto Alegre: Artes Médicas.

Raphael-Leff, J. (2000). Introduction: Technical issues in perinatal therapy. Em J. Raphael-Leff (Org.). 'Spilt milk' perinatal loss \& breakdown (pp. 7-16). Londres: Institute of Psychoanalysis.

Reece, S. M. (1993). Social support and the early maternal experience of primiparas over 35. Maternal-Child Nursing Journal, 21(3), 91-98.

Robinson, G. E., Garner, D. M., Gare, D. J., \& Crawford, B. (1987). Psychological adaptation to pregnancy in childless women more than 35 years of age. American Journal of Obstetrics and Gynecology, 156(2), 328-333.

Rubin, R. (1975). Maternal tasks in pregnancy. Maternal-child Nursing, 4, 143-153.
Soifer, R. (1980). Psicologia da gravidez, parto e puerpério (4 ${ }^{\mathrm{a}}$ ed.). (I. V. de Carvalho, Trad.). Porto Alegre: Artes Médicas.

Stark, M. A. (1997). Psychosocial adjustment during pregnancy: The experience of mature gravidas. Journal of Obstetric and Gynecology Neonatal Nursing, 26(2), 206-211.

Tarin, J. J., Brines, J., \& Cano, A. (1998). Long-term effects of delayed parenthood. Human Reproduction, 13(9), 2371-2376.

Tarin, J. J., Vidal, E., Perez-Hoyos, S., Cano, A., \& Balasch, J. (2001). Delayed motherhood increases the probability of sons to be infertile. Journal of Assisted Reproduction and Genetics, 18(12), 650-654.

Tedesco, J. (1997). Aspectos emocionais da gravidez de alto risco. Em M. Zugaib, J. Tedesco \& J. Quayle (Orgs). Obstetrícia psicossomática (pp. 99-108). São Paulo: Editora Atheneu.

Versellini, P., Zuliane, G., Rognoni, M. T., Trespidi, L., Oldani, S., \& Cardinale, A. (1992). Pregnancy at forty and over: A case-control study. European Journal of Obstetrics \& Ginecology and Reproductive Biology, 48, 191-95.

Windridge, K.C., \& Berryman, J. C. (1999). Women's experiences of giving birth after 35. Birth, 26(1), 16-23.

Winslow, W. (1987). First pregnancy after 35: What is the experience? MCN: The American Journal of Maternal Child Nursing, $12(2), 92-96$.

Woollett, A., \& Marshall, H. (2001). Motherhood and mothering. Em R. K. Unger (Org.). Handbook of the Psychology of Women X Gender (pp. 170-82). Wiley: R. K. Unger.

Zavaschi, M. L., Costa, F., Brunstein, C., Kruter, B. C., \& Estrella, C. G. (1999). Idade materna avançada: Experiência de uma boa interação. Revista de Psiquiatria do Rio Grande do Sul, 21(1), 16-22.

Recebido: $12 / 06 / 2006$

Última revisão: 22/04/2008

Aceite final: $25 / 05 / 2008$

Nota:

${ }^{1}$ No Brasil, os dados disponíveis refletem a realidade da população atendida somente no Sistema Único de Saúde (SUS), o que não corresponde à tendência observada nos países industrializados.

\section{Sobre os autores:}

Aline Grill Gomes: Psicóloga, Mestre e Doutora em Psicologia do Desenvolvimento pela UFRGS e Especialista em Psicologia Clínica pelo IEPP.

Tagma Marina Schneider Donelli: Psicóloga, Mestre e Doutora em Psicologia do Desenvolvimento pela UFRGS e Especialista em Psicologia Hospitalar pela ULBRA.

Cesar Augusto Piccinini: Fez doutorado e pós-doutorado na University College London (Inglaterra), é Pesquisador do CNPq e Professor do Curso de Pós-Graduação em Psicologia do Desenvolvimento da Universidade Federal do Rio Grande do Sul (UFRGS).

Rita de Cássia Sobreira Lopes: Fez doutorado na University College London (Inglaterra) e é Professora do Curso de Pós-Graduação em Psicologia do Desenvolvimento da UFRGS.

Endereço para correspondência: Aline Grill Gomes - Instituto de Psicologia, Universidade Federal do Rio Grande do Sul - Rua Ramiro Barcelos, 2600, sala 111 - 90035-003 - Porto Alegre, RS, Brasil. Endereço eletrônico: algrill7@yahoo.com.br 